Article

\title{
Mapping Transmedia Marketing in the Music Industry: A Methodology
}

\author{
Linda Ryan Bengtsson * and Jessica Edlom \\ Department of Geography, Media and Communications, Karlstad University, Sweden; \\ E-Mails: linda.ryan-bengtsson@kau.se (L.R.B.), jessica.edlom@kau.se (J.E.) \\ * Corresponding author
}

Submitted: 25 January 2021 | Accepted: 29 March 2021 | Published: 5 August 2021

\begin{abstract}
Over the last decade, the music industry has adapted its promotional strategy to take advantage of the fluid, contemporary, platform-based transmedia landscape. For researchers of contemporary culture, the multiplicity of promotional activities creates substantial methodological challenges. In this article, we present and discuss such methodological approaches using two studies of contemporary promotional music campaigns as illustrative cases. Inspired by digital and innovative methods and guided by the Association of Internet Researchers' (AolR's) ethical guidelines, we developed two data collection strategies - reversed engineering and live capturing-and applied two analytical approaches-visual mapping and time-based layering. The first case study traced already staged music marketing campaigns across multiple online media platforms, and the second followed an online promotional campaign in real time for six months. Based on these case studies, we first argue for the importance of grounded manual capturing and coding in data collection, especially when working around data access limitations imposed by platforms. Second, we propose reversed engineering and live capturing as methods of capturing fragmented data, in contemporary promotional campaigns. Third, we suggest the visual mapping and time-based layering of data, enabling researchers to oscillate between qualitative and quantitative data. Finally, we argue that researchers must pool their experiences and resources regarding how to transcend platform limitations and question a lack of transparency while respecting ethical norms and guidelines. With these arguments, we assert the researcher's necessary role in understanding and explaining the complex and hybrid contemporary promotional landscape and provide tools and strategies for further research.
\end{abstract}

\section{Keywords}

digital methods; engagement; ethics; innovative methods; music industry; promotional culture; transmedia marketing

\section{Issue}

This article is part of the issue "Complexity, Hybridity, Liminality: Challenges of Researching Contemporary Promotional Cultures" edited by lan Somerville (University of Leicester, UK) and Lee Edwards (London School of Economics and Political Science, UK).

(C) 2021 by the authors; licensee Cogitatio (Lisbon, Portugal). This article is licensed under a Creative Commons Attribution 4.0 International License (CC BY).

\section{Introduction}

Over the last decade, the music industry has adapted a specific promotional strategy to take advantage of the fluid and dynamic contemporary platform-based media landscape. Such transmedia campaigns are continuously in motion and carried out with significant crossover between online and offline activities with multiple platforms and venues. The term 'transmedia' relates to transmedia storytelling, referring to Jenkins' (2006) concept of how narratives expand and are co-created with audi- ences across different media forms. Transmedia marketing refers to promotional campaigns using a range of media formats and activities to provide a coherent marketing narrative that is co-created with an audience (Scolari, 2009; Zeiser, 2015). Contemporary digital campaigns are often strategically planned to engage audiences across diverse platforms with the intention to generate market value (cf. Jenkins et al., 2013). This allows diverse actors (e.g., advertisers, platform owners, content distributors) to profit from users' activities, i.e., so-called free labour (cf. Hesmondhalgh, 2010; Morris, 
2014; Terranova, 2000), but it simultaneously offers people experiences of community, belonging, and joy (Baym, 2018; Duffett, 2013). Promotional transmedia music campaigns are such hybrid spaces of online and offline practises mixing commercial and cultural expressions to form materialised instances of promotional culture.

However, for researchers of promotional culture, the multiplicity of promotional activities across diverse media outlets creates substantial methodological challenges. The wide range of content and media formats (e.g., shares, likes, written text, memes, visuals, hashtags, emojis, videos, apps, games and animations, in any combination), the distance between qualitative elements and quantitative data strands (from punctuation to thousands of posts and reposting across platforms), and grasping and selecting among the wide range of data scraping and analyse tools accessible can be overwhelming (Rieder \& Röhle, 2017). These challenges are multiplied by the assertion of proprietary control by platforms over online-generated data (Gillespie, 2018). We argue that, in order to pose a wider range of questions about online promotions and the relations between audiences, cultural producers, and platforms, we must be innovative in both our data collection and data analysis methods. New methods may involve skating close to the edge of the platform terms of service, and, as a field, we must thoroughly try to understand the ethical challenges posed by proprietary restrictions placed on social data. To understand a media landscape in flux therefore calls for innovative and sometimes unconventional methods (see, for example, Eriksson et al., 2019).

This article addresses this call by developing methodological strategies for capturing complex promotional campaigns across different media formats as well as how to analyse fragmented qualitative and quantitative data of different formats, forms, and scales and collected from different platforms, all of which have specific affordances and conditions for data collection. The methodological strategies draw on knowledge gained from two largescale case studies on transmedia campaigns within the music industry. Inspired by innovative (cf. Kubitschko \& Kaun, 2016) and digital methods (cf. Pink et al., 2016; Rogers, 2013; Venturini et al., 2018), and guided by the Association of Internet Researchers' (AolR's) ethical guidelines for internet research (Franzke et al., 2020), we developed two data collection strategies, reversed engineering and live capturing, and applied two analytical approaches, visual mapping and time-based layering. We will argue that reversed engendering and visual mapping are applicable when tracing and analysing already staged promotional campaigns and that live capturing and time-based layering are beneficial when studying ongoing campaigns. We will use two case studies of music marketing campaigns to illustrate our developed methodological strategies. The reason for this is twofold: First, inspired by Pink et al. (2016), these cases can be understood as templates for future studies as well as give insight into promotional campaigns' entanglement with a variety of media platforms and user practices. Second, the cases demonstrate the complexity in collecting and navigating diverse strands of data as well as any challenges emerging throughout the study that demand innovative measures.

This article initially accounts for the growing body of work on digital methods and innovative methods. Thereafter, we account for the grounded and iterative processes of refining our methodological strategies. The following section discusses the practical and ethical challenges of identifying and applying methods when studying contemporary promotional campaigns. In the concluding section of the article, we formulate four methodological strategies that we argue will aid researchers in conducting future studies on promotional transmedia campaigns.

\section{Digital and Innovative Methods}

The shift from network-based web structures to the platformisation of communication (Arvidsson, 2016; Helmond, 2015; Van Dijk, 2012) has elevated and enabled the use of digital data as scientific data, especially in regard to data produced through social media platforms, such as Facebook, Twitter, Instagram, and YouTube (McCay-Peet \& Quan-Haase, 2017). This development has called for new methods of capturing, handling, and analysing digital data in a transparent and replicable way. This has prompted interdisciplinary approaches bridging social and computational sciences and the combination of techniques from computer science and theories from social sciences (Tinati et al., 2014), resulting in a range of qualitative and quantitative methods as well as tools for data collection and visualization. These methods are specifically engaged with ways in which to capture and analyse online content; however, their intention is not to study internet culture per se, but rather for social and cultural research. Rogers (2013) therefore formulates digital methods as a 'research practice that learns from the methods of online devices, repurposes them, and seeks to ground claims about cultural change and societal conditions in web data' (p. 19). The study of digital data-or traces of actions - can then be twofold: to study the actions themselves as part of the specific phenomenon studied or as proxies of other actions outside the medium (Venturini et al., 2018).

There is a growing body of guidelines and handbooks for digital methods of conducting, sampling, collecting, and storing data (cf. Hurwitz et al., 2018; Sloan \& Quan-Haase, 2017; Venturini et al., 2018). These guidelines are also a consequence of a range of implications that challenge empirical investigations through digital methods, such as data richness, limitations in data access, policies, privacy issues, and algorithms (cf. Gerlitz \& Rieder, 2013; Gillespie, 2018; Rieder \& Röhle, 2012). Platforms are gatekeepers determining the scope and quality of accessible data and may even deny data access (Gerlitz \& Rieder, 2013; Venturini et al., 
2018). Data is formatted to favour specific types of analysis beneficial to the platform, such as users' networks, actor influence, and content reach, which limit the questions the data can answer (Gitelman, 2013; Marres \& Gerlitz, 2016). Also, determining how algorithms, for example, sort, filter, relate, recommend, and organise content on platforms and search engines is often difficult for researchers (Bucher, 2016; Gillespie, 2018). Instead, researchers need to use unconventional methods to investigate platforms in depth (see, for example, Eriksson et al., 2019, exploring the music distribution platform Spotify). Guidelines have also been developed to ensure the ethical handling of data (cf. Ess, 2013; Zimmer \& Kinder-Kurlanda, 2017). Ethical considerations are always central when conducting research. However, within online research, there are certain additional ethical aspects that need to be taken into account. Accessibility in digital data has also prompted political discussions, resulting in policies and laws (for example, Regulation of 27 April 2016, 2016, within the EU) that need consideration. One of the most thorough guidelines for ethical decision-making while conducting research was developed by the AolR through their Ethics Working Committee (reports 2002, 2012, and 2020). Their most recent report (Franzke et al., 2020) focuses primarily on ethical pluralism and cultural awareness and emphasizes that ethics should be oriented around critical questioning rather than rules. When collecting online data, ethics needs to be addressed throughout the process, from the first initial research design to the end of the project. It demands a bottom-up approach, as there are new considerations to take into account with each case (Franzke et al., 2020).

Digital methods include both qualitative and quantitative methods; however, digital methods have been accused of being biased towards quantitative data (big data), especially because many digital methods borrow methods and analytical tools from computational sciences (further discussed by, for example, Venturini et al., 2018). It is also within the area of extracting and analysing grand volumes of data that we have seen significant development in supportive tools. However, researchers have raised concerns regarding the risk of these tools being applied without substantial understanding of the analytical rationale behind methods rendered in software or seeing its full potential (Rieder \& Röhle, 2017; van Geenen, 2020). A consequent critique of digital methods has been that they have a tendency to be not research-driven, but data-driven (cf. Tinati et al., 2014), where the research question is defined through what different tools can do rather than a specific research question. However, Kitchin (2017) argues that digital methods allow researchers to find new insights that arise within the data rather than through theory, promoting a grounded approach which Rogers (2013) calls 'online groundedness.' Along with Kitchin (2017) and Rogers' (2013) arguments, we want to emphasize the grounded qualities of working with digital methods.
The complexities of handling different interfaces, affordances and data accessibilities, and asynchronous timelines and data formats call for a grounded and adaptive approach (Lury \& Wakeford, 2012). We call this 'being with the data' to bring attention to the necessity of being responsive, adaptive, and grounded while collecting and handling digital data.

When researching contemporary promotional transmedia campaigns, digital methods are close at hand, as they provide guidance and tools for data collection and analysis. Carah and Angus (2018), for example, used quantitative data gathering to capture branded activities on Instagram through hashtags, and Kjus (2016) used quantitative data from WiMP/Tidal to investigate how music is discovered on streaming platforms. Both these studies discuss the role of strategies and algorithms in orchestrating activities to promote participation. Both studies also complemented digital quantitative studies by using qualitative data collection, specifically highlighting the need for different types of data to study the interconnections between media platforms' infrastructures, cultural experiences, cultural consumption, and promotion practices. The most prominent digital qualitative method is digital ethnography, which offers guidance in studying digital social spaces and digital practises embodied in the everyday (Hine, 2015; Pink et al., 2016). Digital ethnography as a method and process offers an explorative, adaptive, and reflexive approach, where the research design and selection of methods should evolve if necessary as the study progresses (Hine, 2015; O'Reilly, 2012; Pink, 2016). Digital ethnography is applicable when, for example, locating and exploring online brand communities. For example, Arvidsson and Caliandro (2016) applied digital ethnography to perform a qualitative analysis of a large twitter data set when studying brand-related communication across social media. Armstrong et al. (2016) examined the innovative marketing practices of the Los Angeles Kings hockey organization and fans' reactions to it. Chang and Park (2019) used digital ethnography to examine the fan club of the music group BTS and its social media activities in connection to its brand. The method repertoire of digital ethnography includes activities such as observations, taking part in and recording activities, interviewing informants, distributing questionnaires, gathering data and artefacts, mapping and visualizing data, and interpreting results. Digital ethnography is a matter of engaging with the field, watching what happens, and listening to what is said (Hine, 2015; O’Reilly, 2012; Pink, 2016).

Kubitschko and Kaun (2016) suggest that researchers need to allow themselves to apply innovation to 'widening and rethinking research methods to further understand the role that media technologies and infrastructures play in society' (p. 4), applying a more pragmatic approach to conducting research in the contemporary media landscape (see also Lury \& Wakeford, 2012; Snee et al., 2016). Innovative methods acknowledge the complexity of following pre-defined methodological paths 
when studying ever-changing contemporary media phenomena. Innovative methods allow researchers to explore methods-by both extending existing methods and developing new methods-while conducting their research (Kubitschko \& Kaun, 2016). The innovative methods initiative allowed for the combining of digital methods with other methods as well as analytical system re-framing methods to be adjusted according to the research question. The ability to be sensitive to the case is crucial when studying digital promotional campaigns (see also Brodmerkel \& Carah, 2016; Lury, 2009). Digital promotional campaigns are staged to move across different platforms and between offline and online activities. Brembilla (2019) studied artist Beyoncé to illustrate how, for example, a matrix of narratives are formed using multiple platforms that, in the end, point to content streaming. Cross-platform data collection, as discussed by Venturini et al. (2018), makes data collection even more complicated, as it involves taking the nature of different platforms into account and simultaneously identify a consistency to enable a common analytical frame. Each platform has different limitations and possibilities regarding the capturing of data, and there is always a risk that the platform will shift its conditions or not provide data. Limited data access prompted, for example, Eriksson et al. (2019) to use an interventionist research design to study the music streaming service Spotify, which led to Spotify claiming that the researchers violated their terms of use (Eriksson et al., 2019). Online campaigns often - if not always - intersect promotional content with user-generated content and individual (media) practices, prompting research questions that integrate qualitative and quantitative data. In addition, as Brodmerkel and Carah (2016) conclude, management and brands often analyse user-generated digital data to experiment with and calibrate their campaigns according to users' activity and responses. This indicates that we, as researchers, need to be innovative alongside the innovative campaigns we examine.

\section{Two Methodological Approaches for Studying Digital Promotional Transmedia Campaigns}

The following section of the article will present and discuss methodological approaches to studying promotional transmedia campaigns. Unfolding of campaigns aims to investigate a wide range of issues of promotional culture, such as engagement, promotional strategies, co-creation, fan/brand cultures, blurred boundaries between professional and unprofessional content, content circulation, transmediality, ethics, and power relations. However, while campaigns are interesting sites of studies, the complexity of how digital campaigns unfold across diverse media outlets and platforms control over online-generated data makes such studies methodologically challenging. In the following section, we propose two different ways of capturing and organising data when studying digital promotional transmedia campaigns. The first section addresses how to approach already conducted campaigns and re-create the outline of a campaign in time and space. The second approach involves the real-time capturing of a promotional campaign as making suggestions of how to organize and analyse large strands of qualitative and quantitative data of different formats.

\subsection{Reversed Engineering and Visual Mapping}

In this study, we aimed to scrutinize how promotional transmedia campaigns play out within the online environment and capture audiences' reactions, specifically campaigns that sought new grounds with online marketing and employed live events and social media platforms in addition to traditional media coverage. Evidence of audience engagement is for example, web traffic, visitors, shares, likes, downloads, uploads, etc. The methodological challenge was to gather data associated with promotional campaigns that had already taken place. One suggested approach is to use existing data collecting tools for scraping platforms and websites of data (McCay-Peet \& Quan-Haase, 2017). However, studying a range of differently designed campaigns using various media platforms demands the use of different tools depending on platform. In addition, we risked gathering an overwhelming amount of data-and data in different formats-which would make it difficult to handle and analyse. Instead, inspired by Suchman's (2012) notion of 'reversed engineering,' we decided to manually trace how each campaign unfolded. Suchman (2012) suggests that the relationships between technology and users (machines and humans) are configurations. By conducting reversed engineering, researchers can understand the components of such relationships and how relations are constructed (Suchman, 2012). When approaching a transmedia marketing campaign as a configuration, reversed engineering brings specific attention to the components and construction of engagement. We thereby reversed the campaigns, capturing the campaigns different types of activities, where those activities took place, and how they were constructed as well as how the campaign was laid out by its management across platforms, events, and time. Reversed engineering allows for gathering data traces of audiences' presence (or absence) and their activities to indicate indifference toward, for example, conducting interviews with management. As a result, we were able to study their actions to understand their nature as well as the proxies of the management's strategies (Venturini et al., 2018). Furthermore, we were able to see and access the same actions as the audience/consumers-or, in other words, be with the data.

\subsubsection{Data Collection and Sampling}

The reversed engineering process became, in many ways, a manual exercise. The report description gave us a point 
of entry into each campaign-for example, a website, YouTube clip, or Facebook address. We took screenshots of the websites, platform activities, and other media outlets to which the campaign directed us. We followed trails that ran from online spaces to offline activities or from virtual to physical places (e.g., from Facebook to a festival site or a radio show to a campaign site and then a streaming service). Following these traces across diverse platforms, we captured what kinds of actions and interactions consumers were asked to undertake (e.g., sharing, liking, contributing content, etc.), as well as the 'promises' that those actions hold for themthat is, what they are offered in return for their (interlactions (e.g., 'exclusive' content, a prize, the ability to impact the order of songs appearing on an album, etc.). Simultaneously, we described each event (e.g., 'the user was asked to follow a link and a hashtag that directed them from Instagram to iTunes') to capture the connection between different media outlets and events. The intention was to ensure that the activity was captured within its context, especially as most of these activities were combinations of text, images, and responses. Finally, the data gathered consisted of screen shots of different campaign activities and audience responses accompanied by textual descriptions of each activity and response.

\subsubsection{Visual Mapping for Data Analysis}

The next step was to identify a way to organise the collected data. Returning back to how reversed engi- neering is formulated by Suchman (2012), there was a need to re-configure the relationship between technology and users. The descriptions of each activity combined with screenshots facilitated a recreation of the campaigns activities and movement across platforms. Each campaigns' activities could be mapped out in relation to each other modelling a visual map of the whole campaign. An example of a part of a campaign map is presented in Figure 1 below. The campaign starts from the top right and then evolves following the arrows. The arrows were given different colours to separate campaign activities and fan responses. Constructing a visual map of campaigns allows for a reconstruction of activities, comparison between campaigns, identification of the relation between the campaign activities and audience responses, and organising activities in time and place (e.g., platforms, another media outlet, or off-line). Stieglitz et al. (2018) point out that visualization of data should not only be a way to communicate results but should also be integrated into the research process. This was not a clear intention when we defined our approach to gathering data, but it became central to our discovering and organizing topics and events relevant to our research question and then explore them further. By mapping activities, we identified how users move through the campaign's time-space and how the campaign prepared trails to guide the consumer to various media platforms (from an official website to Instagram, Spotify, etc.). The map then described how the campaign narrative unfolded and identified interconnections and collaborations with other organizations and events,

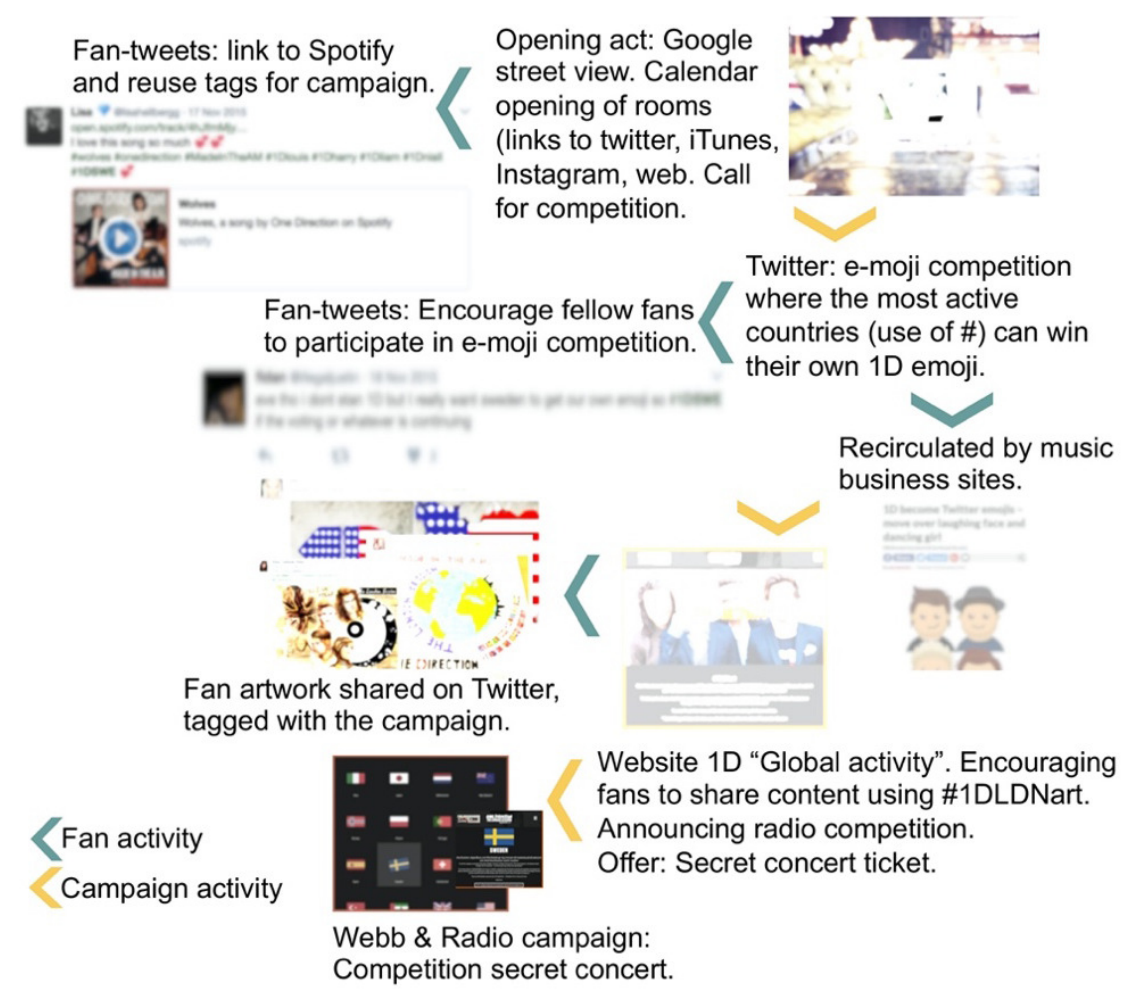

Figure 1. An example of a visual map for the One Direction marketing campaign. 
for example, how boyband One Direction built partnerships with Google Street View, Twitter, iTunes, and local radio channels around the world for audience engagement activities as part of their campaign (Figure 1). Hand (2017) emphasises the importance of contextualising activities (especially in regard to images), as they are situated in a complex combination of users, devices, platforms and produced and consumed with different purposes. Mapping campaign enabled the realization of these complexities. Specifically, mapping through screenshots and observational descriptions served in this case to examine the transmediality within these campaigns. It also gave evidence of transspatiality, recognizing that consumers' mobility also potentially stretches beyond the media. Constructing a map thereby visualised a 'transmediascape' interlinking presumption and free labour and suggesting that users are 'workers' cultivating the digital soil along a controlled path across the transmediascape (Fast et al., 2017; Fast \& Jansson, 2019).

\subsubsection{Challenges and Limitations of Reversed Engineering and Visual Mapping}

Using reversed engineering to gather data and reconstructing campaigns using visual mapping offer researchers a way to study already conducted campaigns. However, there are also limitations to this method. Specifically, there is an overwhelming risk when reversing a campaign that the data has been tampered with (Gerlitz \& Rieder, 2013). Platforms as well as the campaign management-or even users - could have curated the content. Following a completed marketing campaign also means that researchers can only capture activities incorporated in the campaign-not any live changes made to it, only traces of the audience's reaction to the campaign in real time. Fan activities and responses to a campaign may occur in other forums or platforms that are not interlinked with the campaign. In that regard, the starting point of the reversed engineering is crucial to the data gathering and mapping enfolds. However, reversed engineering combined with visual mapping offers a richness in data and a grounded approach to how promotional transmedia campaigns unfold.

\subsection{Live Capturing and Time-Based Layering of Data}

While above we have engaged with the methodological challenges of studying an already conducted campaign, the following section will focus on how to methodologically approach an ongoing transmedia campaign. The intention is to investigate how a transmedia marketing campaign plays out in real time. Although backtracking completed campaigns offers valuable insights into how a transmedia campaign unfolds, we here seek to identify ways to capture audiences' reactions to campaign activities more accurately. In this section, we therefore address a way with which to follow a specific promotional campaign, capturing the audience's actions and interactions, and do so in relation to how the campaign moved across a transmediascape in time and space. Again, we use a specific case study as an illustrative case, a promotional campaign staged in conjunction with the release of Taylor Swift's album Reputation. The case illustrates the complexity of contemporary transmedia campaigns at the intersection of cultural expression, branding, and promotion as well as how a campaign is not a standalone entity but highly integrated with previous campaigns and dialogs with audiences/fans.

\subsubsection{Data Collection and Sampling}

Approaching a promotional campaign demands an initial recognition of the object of study's current platform presence. In this case, we identified official accounts on Twitter, Instagram, Facebook, and YouTube. On Twitter, Instagram, and Facebook, we also detected a double presence: one personal account and one management account. These, along with an official website and presence on iTunes, formed the basis of the campaign. However, during the study, the campaign also entered new platforms, in this case the social media platforms Tumblr and TikTok, in addition to launching a standalone proprietary app. This highlights the importance of a data collection approach with the ability to adapt to the course of the campaign.

There are existing tools for abstracting data from, for example, Facebook, Twitter, and YouTube; however, there are as noted above limits to the access of data and some platforms prohibit data access (Gerlitz \& Rieder, 2013). Capturing a campaign with such extensive use of social media platforms therefore demands a variety of approaches to collecting data. As access and policies shift over time, researchers are often dependent on the tools accessible at the time of the study. In addition, there is the risk that access to data changes during the course of a study. During the Taylor Swift campaign study, Instagram shut down their Application Programming Interface (API), prohibiting us from retrieving data from the platform. Such an uncertainty of data access suggests the necessity of a multi-approach to ensure rigid data collection. In the Taylor Swift case, we used the Twitter data collection tool Mecodify (Al-Saqaf, 2016). Mecodify captures twitter data following hashtags defined by the researcher and creates a timeline over published twitter posts. In this case, we also used downloaded data from Swift's Facebook account using Netvizz (Rieder, 2013). Netvizz captures posts as well as likes, shares and comments associated with the post and associated with the specific users. It also collects the location of the userif accessible. We also collected data from Taylor Swift's YouTube account through a transcript scraper, collecting video posts, comments, likes, and shares associated with a specific account (Rieder, 2015).

The gathered data offered insights into activity and individual user data; however, one should not neglect the importance of how content appears and is organized 
within the interface of a platform. The interface also offers an overview of how dialogues and relationships emerge between activities. Most importantly, it also represents with what the user is confronted as well as how the campaign aims their activities to be seen. Therefore, along with scraping data, we observed the campaign as it developed by capturing screenshots of different events that took place throughout and followed up with links or activities posted by the campaign, especially those interlinking with other platforms. Furthermore, we solved the problem of the closure of Instagram's API by taking screenshots of posts and user responses throughout the campaign. We therefore manually followed Swift's official Instagram accounts (@taylorswift and @taylornation) throughout the study. This became crucial to the study when the campaign moved to other platforms that we could not access through scraping. Real-time observations of ongoing practices allowed us as researchers to, at least partially, transcend the data access limitations set by the platforms (Gerlitz \& Rieder, 2013) and capture data before it was (possibly) cleaned by the campaign.

\subsubsection{Time-Based Data Layering for Analysis}

When live sampling a campaign, it is difficult to foresee its duration and course. After only a few weeks of sampling, and still before the actual release of the album, we had already gathered large amounts of data (quantitative data using scraping tools and qualitative data consisting of screen shots), and we realized that we had to start analysing our data in parallel with data collection. Using the data from the first six weeks of the campaign, we explored different types of analytical approaches. We used Gephi to conduct a network analysis of Swift's Facebook account, which indicated her position as a hub in the music industry social media network. We also explored visualizations of different types of data (e.g., location of users); however, it was difficult to draw any further conclusions from this. With limited insight into the analytical tools' functions and handling of the data (Rieder \& Röhle, 2017), it proved to be difficult to ask the questions we wanted to of the data. Instead, it was when we explored diagrams of likes, shares, and comments from the Facebook data as well as a diagram of Twitter activity that we started to see patterns and shapes (Figure 2). We overlayered the Twitter data with the Facebook data and could see that they were interrelated over time. Then, we organized our qualitative data along a timeline, visually over-layering this qualitative data with the quantitative data (see example below). We could then oscillate our analysis between a macro and micro level. Layering the data illustrated the interconnectedness between the campaign activities and audience engagement and highlighted specific

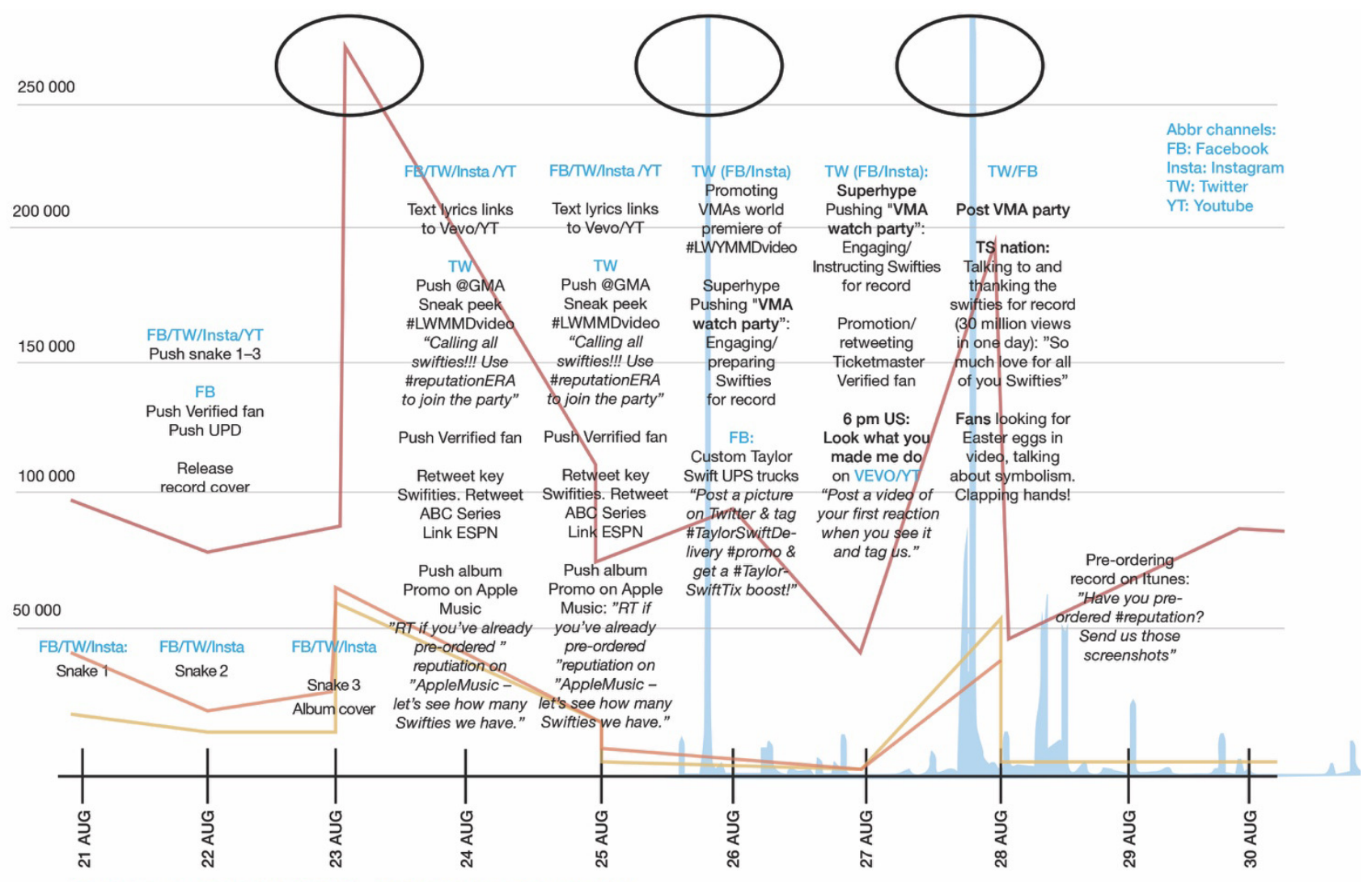

Figure 2. Example of visual layering of qualitative and quantitative data in regard to specific campaign activities and layering data from Facebook, Twitter, Instagram, and YouTube. 
events that require further study. One example was a post that was posted both on Twitter and Facebook that was largely shared and liked. The post contained only three images and, unlike most of the other posts, had no additional text, links, or hashtags. To attempt to understand the audience's excitement, we followed up on several of the Facebook accounts that had liked and shared this image. In doing so, we could identify that these accounts had shifted their Facebook headings and account images using the visual expression of the upcoming album. Conducting this form of qualitative content analysis allowed for 'categories to emerge out of data and on recognizing the significance for understanding meaning in the context in which an item being analysed (and the categories derived from it) appeared' (Bryman, 2012, p. 291). Performing content analysis, especially regarding studying media-rich content, comes with certain challenges (e.g., collecting and handling ever-changing media formats; see, for example, Hurwitz et al., 2018); however, this study benefitted from reformatting the data to allow for coding and synthesizing it more deeply.

At this point, we realised that we would not be able to handle the overwhelming amount of data collected through the scraping tools. Instead, we needed to identify a more effective way to gather data. After the first analysis, we revisited our research question, which directed our efforts to specifically focusing on instances of cross-platform and offline activities. Performing data collection and analysis in parallel here proved central to focusing on data gathering along with the research question. Our findings showed a close interconnection between fan culture and promotion. In our study we found that the music industry feeds fan culture events around established music events (e.g., the MTV Video Music Awards), and mobilizes to take control of audience participation by playing into existing fan expressions, making use of platforms' affordances and actively seeking audiences on new platforms. The manner in which to participate is defined by the industry and echoed and amplified among fans.

\subsubsection{Challenges and Implications of Live Capturing}

Following a global marketing campaign in real time can come with several challenges. The amount of data that can be collected is almost endless, and there are many considerations and decisions to make during the process. Such considerations include which platforms to follow and during which time period and time zone they should be followed, what to do with the enormous amount of data that is collected, how to know whether the data collected is the right data and that some central aspects have not been missed, and how to store the data in a way that is feasible in terms of both storage space/place and ethical aspects. When live capturing, these decisions are the researchers' responsibility and can involve possible dead ends. However, live capturing can also facili- tate being open and explorative during the process, as the route is not decided in advance. This way of examining is suitable for a promotional cross-media campaign, which in itself also needs to be iterative and follow and adapt to audience engagement-the campaign in itself is evolving during the process, and so is the research to be conducted.

\section{Ethical Challenges}

Capturing promotional transmedia campaigns across different media formats poses many new challenges for researchers-not in the least of which are ethical challenges. During our studies, we followed the AoiR Ethics Working Committee guidelines for internet research (Franzke et al., 2020), and several ethical issues were considered, especially those concerning data storage, privacy issues, and the visualization/representation of our findings. Researching over a more extended period and working across platforms added to the complexity to data storage. Scraping tools for established platforms (Twitter and Facebook in this case) collect data onto a defined server, limiting external access. However, visiting different platforms and conducting manual data collection makes it complicated (or even impossible) to maintain control of the data. When following the guidelines for data storage from the AolR (Franzke et al., 2020), special measures are required to store data in a secure, disconnected way. However, live capturing a campaign makes this quite difficult to accomplish. For example, several apps often used in campaigns are only accessible through a mobile phone. In retrospect, this activity should have been conducted in a more systematic and controlled way and on specific devices during our study. However, we were unable to foresee this development in the campaign. The measures we did take included focusing only on specific activities, in agreement with the rule of data minimization as per the General Data Protection Regulation (Regulation of 27 April 2016, 2016), and only collecting data relevant to the research question.

In both the studies, we followed links and captured data partially by taking screenshots, as described above. In a way, we were 'lurkers,' observing ongoing activities and conducting observations without users consent. Observations are not necessarily problematic as such (as discussed by Murthy, 2008); However, the screenshots visually depict individuals' somewhat private activities and expressions. Screenshots are beneficial for analysis, as they are representations of ongoing activities at a particular time. Also, in this case, we can assume that the social media feeds are controlled and possibly tampered with by, for example, the management team in addition to the platform's own monitoring and manipulation of content (for further discussions on the limitations of data scraping, see, for example, Gerlitz \& Rieder, 2013). With uncertainty regarding to what extent we can rely on the data provided from a commercial platform, as suggested by Karlsson (2012), screenshots can 
then represent what is published-at least at that specific moment. However, working with visuals in a digital context is a sensitive matter. We as researchers give individuals' (inter)actions meaning through our analysis. Sensitivity pertains not only to the topic of study (e.g., sexual preferences, political affiliation, mental health or other medical issues) but also to the nature of the study itself. In our illustrated cases above, we used screen shots to critically engage with user practises without their consent. Following the ethics outlined by the AolR Ethics Working Committee (Franzke et al., 2020), it is therefore of great importance to not use specific examples or dialogues nor show content that could be traced online in academic texts. However, the concept of anonymity partially contradicts our willingness to be transparent. It prohibits us from fully accounting for our visual mapping and overlying of data, as posts and comments need to be neutralized.

\section{Conclusions: Adaptation and Openness by Being with Data}

We propose two approaches to data collection and ways to sort and analyse large strands of diverse data. We thereby want to bring attention to alternative and grounded ways to access, gather, combine, and analyse diverse strands of data. Reversed engineering can be applied to trace already conducted transmedia marketing campaigns across multiple media platforms, on campaign websites, and in web-based games and apps. The collected data can then be organized in time and space through visual mapping, thus re-creating the campaigns for analysis. Live capturing enables researchers to follow a promotional campaign in real time and over time as well as across promotional material, concerts, and events mixing both offline and online audience-targeted and audience-generated activities. Moreover, live capturing generates qualitative and quantitative data of different formats and scales, which can then be interconnected, structured, and analysed using time-based layering.

Throughout our work, it became obvious that conducting online research demands openness and adaptation to the situation grounded in the case (or cases). While the need to be adaptive and innovative is strongly articulated within digital as well as innovative methods, we wanted to emphasize the notion of 'being with the data.' It is key to not get lost in the data, to be responsive to new turns and activities, and to avoid being distracted from the issue at hand. We therefore argue that studying online content is not only a scraping issue; manual capturing and coding can be just as useful for the purpose of such a study, as it allows the researcher to be adaptive and grounded. While it might demand a greater amount of work, manual capturing and coding also might also bring the analysis closer to the data. Moreover, it also allows researchers to engage with rich data sets without being dependent on complex analytical tools. In line with Bucher (2016) and Eriksson et al. (2019), we argue that it becomes the researcher's task to restructure how platform logic plays into the shaping of society. These methodological approaches offer ways to penetrate the limitations and specifications of platforms in order to examine such phenomena in depth.

Based on the methodological approaches presented above, we first argue that manual capturing and coding have an important function in data collection-both alone and in combination with technical processes, such as scraping and using complex analytical data tools. Manual processes are especially important when working around data access limitations imposed by platforms and when handling diverse data formatting. Second, we propose two ways to conduct manual data collectionreversed engineering and live capturing or capturing fragmented data as in contemporary campaigns. Combining manual capturing and scraping ensure data richness and facilitate the contextualisation of campaign activities (Hand, 2017). Third, we suggest the visual mapping and time-based layering of data, as this enables researchers to bring together data of different formats as well as oscillate between qualitative and quantitative data in the analysis. As a part of the innovative methods approach (Kubitschko \& Kaun, 2016), the researcher can oscillate between collecting data, organizing it, and analysing it and collecting more data in new ways. This is an iterative and analytical process that is not set from the start but changes throughout the researcher's work. We therefore want to emphasise the importance of conducting data collection and analysis in parallel, as analysis gives insight into the strategic changes of the study design.

Finally, researchers must pool their experiences and resources on how to transcend platform limitations and question platforms' lack of transparency while respecting ethical norms and guidelines. New methods may involve skating close to the edge of platforms' terms of service, and, as a field, we must also understand the ethical challenges posed by proprietary restrictions on social data. With these arguments, we assert the researcher's necessary role in understanding and explaining the complex and hybrid contemporary promotional landscape and provide further tools and strategies for researching the embeddedness of promotion in everyday life.

\section{Acknowledgments}

The study has been conducted as a part of the research project Music Ecosystems Inner Scandinavia, funded by the Interreg Sweden-Norway programme, EU, and Karlstad University, Inland Norway University of Applied Sciences, Innlandet Fylkeskommune county council, Arvika municipality and Region Värmland.

\section{Conflict of Interests}

The authors declare no conflict of interests. 


\section{References}

Al-Saqaf, W. (2016). Mecodify: A tool for big data analysis \& visualization with Twitter as a case study. Stockholm University.

Armstrong, C. G., Delia, E. B., \& Giardina, M. D. (2016). Embracing the social in social media: An analysis of the social media marketing strategies of the Los Angeles Kings. Communication \& Sport, 4(2), 145-165.

Arvidsson, A. (2016). Facebook and finance: On the social logic of the derivative. Theory, Culture \& Society, 33(6), 3-23.

Arvidsson, A., \& Caliandro, A. (2016). Brand public. Journal of Consumer Research, 42(5), 727-748.

Baym, N. K. (2018). Playing to the crowd: Musicians, audiences, and the intimate work of connection. New York University Press.

Brembilla, P. (2019). Transmedia music, the values of music as a transmedia asset. In M. Freeman \& R. Rampazzo Gambarato (Eds.), The Routledge companion to transmedia studies (pp. 82-89). Routledge.

Brodmerkel, S., \& Carah, N. (2016). Brand machines, sensory media and calculative culture. Springer.

Bryman, A. (2012). Social research methods. Oxford University Press.

Bucher, T. (2016). Neither black nor box: Ways of knowing algorithms. In S. Kubitschko \& A. Kaun (Eds.), Innovative methods in media and communication research (pp. 81-98). Palgrave Macmillan.

Carah, N., \& Angus, D. (2018). Algorithmic brand culture: Participatory labour, machine learning and branding on social media. Media, Culture \& Society, 40(2), 178-194.

Chang, W., \& Park, S. E. (2019). The fandom of Hallyu, a tribe in the digital network era: The Case of ARMY of BTS. Kritika Kultura, 32, 260-287.

Duffett, M. (2013). Introduction: Directions in music fan research: Undiscovered territories and hard Problems. Popular Music and Society, 36(3), 299-304.

Eriksson, M., Fleischer, F., Johansson, A., Snickars, P., \& Vonderau, P. (2019). Spotify teardown inside the black box of streaming music. MIT Press.

Ess, C. (2013). Digital media ethics (2nd ed.). Polity Press. Fast, K., \& Jansson, A. (2019). Transmedia work: Privilege and precariousness in digital modernity. Routledge.

Fast, K., Ryan Bengtsson, L., \& Ferrer Conill, R. (2017). A spatial approach to fan labor: Conceptualizing fan mobilization in transmedia marketing [Paper presentation]. 67th Annual ICA Conference International Communication Association, San Diego, CA, USA.

Franzke, A. S., Bechmann, A., Zimmer, M., Ess, C. M., \& Association of Internet Researchers. (2020). Internet research: Ethical guidelines 3.0. Association of Internet Researchers. https://aoir.org/reports/ ethics3.pdf

Gerlitz, C., \& Rieder, B. (2013). Mining one percent of Twitter: Collections, baselines, sampling. $M / C$ Journal, 16(2). https://doi.org/10.5204/mcj.620
Gillespie, T. (2018). Custodians of the internet: Platforms, content moderation, and the hidden decisions that shape social media. Yale University Press.

Gitelman, L. (Ed.). (2013). Raw data is an oxymoron. MIT Press.

Hand, M. (2017). Visuality in social media: Researching images, circulations, and practises. In L. Sloan \& A. Quan-Haase (Eds.), The SAGE handbook of social media research methods (pp. 13-26). SAGE.

Helmond, A. (2015). The platformization of the web: Making web data platform ready. Social Media + Society, 1(2), 1-11.

Hesmondhalgh, D. (2010). User-generated content, free labour and the cultural industries. Ephemera: theory \& politics in organization, 10(3/4), 267-284.

Hine, C. (2015). Ethnography for the internet: Embedded, embodied and everyday. Bloomsbury Academic.

Hurwitz, L. B., Alvarez, A. L., Lauricella, A. R., Rousse, T. H., Montague, H., \& Wartella, E. (2018). Content analysis across new media platforms: Methodological considerations for capturing media-rich data. New Media \& Society, 20(2), 532-548.

Jenkins, H. (2006). Fans, bloggers and gamers: Exploring participatory culture. NYU Press.

Jenkins, H., Ford, S., \& Green, J. (2013). Spreadable media, creating value and meaning in a networked culture. NYU Press.

Karlsson, M. (2012). Charting the liquidity of online news: Moving towards a method for content analysis of online news. International Communication Gazette, 74(4), 385-402.

Kitchin, R. (2017). Big data-Hype or revolution? In L. Sloan \& A. Quan-Haase (Eds.), The SAGE handbook of social media research methods (pp. 27-39). SAGE.

Kjus, Y. (2016). Musical exploration via streaming services: The Norwegian experience. Popular Communication, 14(3), 127-136.

Kubitschko, S., \& Kaun, A. (Eds.). (2016). Innovative methods in media and communication research. Palgrave Macmillan.

Lury, C. (2009). Brand as assemblage: Assembling culture. Journal of Cultural Economy, 2(1/2), 67-82.

Lury, C., \& Wakeford, N. (Eds.). (2012). Inventive methods: The happening of the social. Routledge.

Marres, N., \& Gerlitz, C. (2016). Interface methods: Renegotiating relations between digital social research, STS and sociology. The Sociological Review, 64(1), 21-46.

McCay-Peet, L., \& Quan-Haase, A. (2017). What is social media and what questions can social media research help us answer? In L. Sloan \& A. Quan-Haase (Eds.), The SAGE handbook of social media research methods (pp. 13-26). SAGE.

Morris, J. W. (2014). Artists as entrepreneurs, fans as workers. Popular Music \& Society, 37(3), 273-290.

Murthy, D. (2008). Digital ethnography: An examination of the use of new technologies for social research. Sociology, 42(5), 837-855. 
O'Reilly, K. (2012). Ethnographic methods: The practice of ethnography (2nd ed.). Routledge.

Pink, S. (2016). Experience. In Kubitschko, S. \& Kaun, A. (Eds.), Innovative methods in media and communication research (pp. 161-165). Springer.

Pink, S., Horst, H., Postill, J., Hjorth, L., Lewis, T., \& Tacchi, J. (2016). Digital ethnography: Principles and practice. SAGE.

Regulation (EU) 2016/679 of the European Parliament and of the Council of 27 April 2016 on the protection of natural persons with regard to the processing of personal data and on the free movement of such data, and repealing Directive 95/46/EC (General Data Protection Regulation). (2016). Journal of the European Union, L 119.

Rieder, B. (2013). Studying Facebook via data extraction: The Netvizz application. Proceedings of the 5th Annual ACM web science conference (pp. 346-355). ACM.

Rieder, B. (2015). YouTube data tools version 1.0 [Computer software]. https://tools.digitalmethods. net/netvizz/youtube

Rieder, B., \& Röhle, T. (2012). Digital methods: Five challenges. In D. M. Berry (Ed.), Understanding digital humanities (pp. 67-84). Palgrave Macmillan.

Rieder, B., \& Röhle, T. (2017). Digital methods: From challenges to Bildung. In T. Schäfer \& K. van Es (Eds.), The datafied society: Studying culture through data (pp. 109-124). Amsterdam University Press.

Rogers, R. (2013). Digital methods. MIT Press.

Scolari, C. A. (2009). Transmedia storytelling: Implicit consumers, narrative worlds, and branding in contemporary media production. International Journal of Communication, 3, 586-606.

Sloan, L., \& Quan-Haase, A. (Eds.). (2017). The SAGE hand- book of social media research methods. SAGE.

Snee, H., Hine, C., Morey, Y., Roberts, S., \& Watson, H. (2016). Digital methods as mainstream methodology: An introduction. In S. Roberts, H. Snee, C. Hine, Y. Morey, \& H. Watson (Eds.), Digital methods for social science: An interdisciplinary guide to research innovation (pp. 1-11). Palgrave Macmillan.

Stieglitz, S., Mirbabaie, M., Ross, B., \& Neuberger, C. (2018). Social media analytics-Challenges in topic discovery, data collection, and data preparation. International Journal of Information Management, 39, 156-168.

Suchman, L. (2012). Configuration. In C. Lury \& N. Wakeford (Eds.), Inventive methods (pp. 62-74). Routledge.

Terranova, T. (2000). Free labor: Producing culture for the digital economy. Social Text, 18(2), 33-58.

Tinati, R., Phillipe, O., Pope, C., Carr, L., \& Halford, S. (2014). Challenging social media analytics: Web science perspectives. Proceedings of the 2014 ACM conference on web science (pp. 177-181). ACM.

Van Dijk, J. (2012). The network society. SAGE.

van Geenen, D. (2020). Critical affordance analysis for digital methods: The case of Gephi. In M. Burkhardt M. Shnayien, \& K. Grashöfer (Eds.), Explorations in digital cultures. Meson Press.

Venturini, T., Bounegru, L., Gray, J., \& Rogers, R. (2018). A reality check (list) for digital methods. New Media \& Society, 20(11), 4195-4217.

Zeiser, A. (2015). Transmedia marketing: From film and $T V$ to games and digital media. Focal Press.

Zimmer, M., \& Kinder-Kurlanda, K. (2017). Internet research ethics for the social age: New challenges, cases, and contexts. Peter Lang.

\section{About the Authors}



Linda Ryan Bengtsson (PhD) is a Senior Lecturer in Media and Communications at Karlstad University, Sweden. She is an interdisciplinary Researcher and her work investigates the relations between creative life, interactivity, media, design and place. Currently her work focuses on the intersection of technology and place-making by studying practices of music listening in everyday life, as well as mediated tourism and the relation between offline and online marketing. Linda is also a member of the interdisciplinary research group Geomedia.

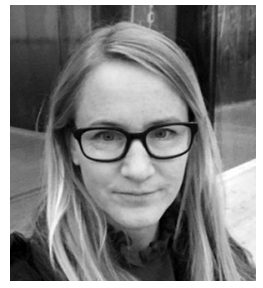

Jessica Edlom is a Doctoral Student in Media and Communication and Adjunct Teacher at Karlstad University, Sweden. She is part of the EU-funded Interreg Sweden-Norway research project "MECOMusic Ecosystems Inner Scandinavia." Her research is focused on strategic communication, communication management and brand building. Jessica's research has appeared in several books and journals including Popular Music, Technology, and the Changing Media Ecosystem and International Journal of Music Business Research. Prior to academia Jessica worked with advertising and strategic communication for both public and private organizations. 